\title{
FOXD1 and Gal-3 Form a Positive Regulatory Loop to Regulate Lung Cancer Aggressiveness
}

\author{
Chien-Hsiu Li ${ }^{1,2}$, Yu-Chan Chang ${ }^{3}\left(\mathbb{D}\right.$, Michael Hsiao ${ }^{3,4, *} \mathbb{C}$ and Shu-Mei Liang ${ }^{1,2, *}$ \\ 1 Graduate Institute of Life Sciences, National Defense Medical Center, Taipei 114, Taiwan; \\ dicknivek@icloud.com \\ 2 Agricultural Biotechnology Research Center, Academia Sinica, Taipei 115, Taiwan \\ 3 Genomics Research Center, Academia Sinica, Taipei 115, Taiwan; jameskobe0@gmail.com \\ 4 Department of Biochemistry, College of Medicine, Kaohsiung Medical University, Kaohsiung 80708, Taiwan \\ * Correspondence: mhsiao@gate.sinica.edu.tw (M.H.); smyang@gate.sinica.edu.tw (S.-M.L.); \\ Tel.: +886-227-871-243 (M.H.); +886-227-872-082 (S.-M.L.)
}

Received: 1 November 2019; Accepted: 25 November 2019; Published: 28 November 2019

check for updates

\begin{abstract}
Dysregulation of forkhead box D1 (FOXD1) is known to promote tumor progression; however, its molecular mechanism of action is unclear. Based on microarray analysis, we identified galectin-3/LGALS3 (Gal-3) as a potential downstream target of FOXD1, as FOXD1 transactivated Gal-3 by interacting with the Gal-3 promoter to upregulate Gal-3 in FOXD1-overexpressing CL1-0 lung cancer cells. Ectopic expression of FOXD1 increased the expression of Gal-3 and the growth and motility of lung cancer cells, whereas depletion of Gal-3 attenuated FOXD1-mediated tumorigenesis. ERK1/2 interacted with FOXD1 in the cytosol and translocated FOXD1 into the nucleus to activate Gal-3. Gal-3 in turn upregulated FOXD1 via the transcription factor proto-oncogene 1 (ETS-1) to transactivate FOXD1. The increase in ETS-1/FOXD1 expression by Gal-3 was through Gal-3-mediated integrin- $\beta 1$ (ITG 31 ) signaling. The overexpression of both FOXD1 and Gal-3 form a positive regulatory loop to promote lung cancer aggressiveness. Moreover, both FOXD1 and Gal-3 were positively correlated in human lung cancer tissues. Our findings demonstrated that FOXD1 and Gal-3 form a positive feedback loop in lung cancer, and interference of this loop may serve as an effective therapeutic target for the treatment of lung cancers, particularly those related to dysregulation of Gal-3.
\end{abstract}

Keywords: lung cancer; FOXD1; Galectin-3

\section{Introduction}

Lung cancer is one of the most prevalent cancers worldwide [1]. Approximately $80 \%$ of lung cancers are non-small cell lung cancers (NSCLCs) [2]. Surgery, chemotherapy, radiotherapy, molecular-targeted therapy, and immunotherapy are the current standard treatments. However, patients with advanced lung cancer have 10-15\% overall 5-year survival rates, an median overall survival (OS) of about 11.1 months, and a post-recurrence survival rate (PRS) of $13 \%$ (hazard ratio $(\mathrm{HR})=0.78$ ) [2-4]. Even with second-line drug treatment, such as nivolumab, progression-free survival (PFS) is about 2.3-3.5 months [5]. It is therefore critical to elucidate the molecular mechanisms of lung cancer proliferation and metastasis to identify and develop novel therapeutic targets in lung cancer.

Transcription factor (TF) dysregulation has been linked to tumor progression, and targeting TFs may be an effective strategy for cancer intervention [6]. FOXD1, also known as FKHL8 and FREAC-4, is a transcription factor that belongs to the forkhead box (FOX) family [7-10]. FOXD1 is associated with cell programming, especially in renal and kidney development [7-10]. Abnormal FOXD1 expression is involved in the progression of tumors [11] including breast cancer [12], colorectal cancer, melanoma, glioma [13], osteosarcoma, renal cell carcinoma, ovarian carcinoma, medulloblastoma [14], and lung 
cancer [15]. Recently, clinical mRNA microarray data identified FOXD1 as a factor associated with poor prognosis and that is required for lung cancer cell proliferation [15]. FOXD1 overexpression is strongly associated with NSCLC proliferation and metastasis through the activation of vimentin [16]. Although several studies have tried to unravel the molecular networks of FOXD1 [11-13,16,17], the molecular mechanisms related to FOXD1 remain largely unexamined, especially in lung cancer.

Gal-3 belongs to the $\beta$-galactoside-binding lectins family [18]; it is ubiquitously expressed in different cellular compartments, including the cytoplasm, nucleus, cell surface, and extracellular matrix $[19,20]$. Gal-3 has pleiotropic functions ranging from gene splicing, cell-cell interactions, and immune responses, to embryonic development [19-22]. Elevated Gal-3 contributes to tumor progression in different human cancers [23-26]. Extracellular Gal-3 acts as a mediator that regulates metastatic cancer processes by recognizing $\mathrm{N}$-acetyllactosamine ( $\beta$-galactoside)-conjugated proteins, including EGFR, integrins, TGF- $\beta$ receptors, etc. $[25,27,28]$. For instance, the interaction of integrin $\beta 3$ and Gal-3 contributes to EGFR tyrosine kinase inhibitor resistance, which facilitates lung cancer cell stemness properties and renders them more resistant to chemotherapeutic agents [29]. The interaction between Gal- 3 and $\beta$-catenin is required for drug resistance and stemness in lung cancer [21]. Therefore, several studies have searched for potential strategies by targeting Gal-3 $[19,25,26,30]$. These studies showed that Gal-3 expression is regulated by bFGF, NGF-mediated MEK/ERK signaling, and various transcription factors, such as Runx family proteins, HIF-1 $\alpha$, and NF-kB [30]. Whether there is any interrelationship between FOXD1 and Gal-3 remains unclear.

In this study, we demonstrated that FOXD1 bound to the Gal-3 promoter and positively transactivated Gal-3 expression. Moreover, ERK1/2 was required for FOXD1 translocation into the nucleus by associating with FOXD1. Notably, the ERK signaling cascade was mediated by the Gal-3/ integrin- $\beta 1$ (ITG $\beta 1$ ) axis. Gal-3 induced FOXD1 expression by associating with the ITG $\beta 1 / F A K / P I 3 K /$ AKT/PHB1/Ras/Raf-1 complex in lipid rafts and increasing proto-oncogene 1 (ETS-1) to transactivate FOXD1. Gal-3 mediated ERK activation, leading to the translocation of FOXD1 into the nucleus to regulate Gal-3 expression. The co-expression of FOXD1 and Gal-3 promoted, whereas the co-knockdown of both attenuated, lung cancer cell growth and motility in vitro. Furthermore, using clinical human lung cancer tissue microarrays, we found that both FOXD1 and Gal-3 were positively correlated in advanced lung tumor tissues.

\section{Results}

\subsection{High FOXD1 and Gal-3 Expression are Associated with the Poor Prognosis in Lung Cancer}

To identify novel downstream molecules involved in FOXD1-mediated lung cancer aggressiveness, FOXD1 cDNA was overexpressed in cells or knocked down by specific siRNA, and mRNA microarrays and comparative gene expression analyses were then performed. We selected the probes in which there was $>5$-fold change in FOXD1 knockdown versus vector control in CL1-0 cells. Using Ingenuity Pathway Analyses (IPA), we identified several potential regulators (Figure 1A). Among these regulators, Gal-3 was shown to be one of the FOXD1 downstream effectors. In addition, the core-analysis from IPA also revealed that RNA level of Gal-3 (LGALS3) was increased by $>100$-fold by FOXD1 upregulation (Figure 1B). Furthermore, both FOXD1 and Gal-3 mRNA and protein levels were increased in lung tumor cells, such as CL1-0, CL1-5, and H1299 cells, compared with normal lung cells (WI-38) by qPCR (Figure 1C) and Western blot (Figure 1D) assays. We also found that a high level of FOXD1 mRNA and protein expression was positively correlated with Gal-3 expression in more aggressive cells, such as CL1-5 and H1299 (Figure S1A,B). We thus evaluated FOXD1 and Gal-3 gene expression in lung cancer patients from the SurvExpress database (LUAD-TCGA and Lung Meta-base) and found high FOXD1 and Gal-3 expression in the high-risk group (Figure 1E). Moreover, lung cancer patients with high FOXD1 and Gal-3 gene expression were associated with a poor prognosis in a different database (LUAD-TCGA and Lung Meta-base) (Figure 1F). Collectively, these data indicated that FOXD1 might promote lung cancer aggressiveness through the upregulation of Gal-3. 

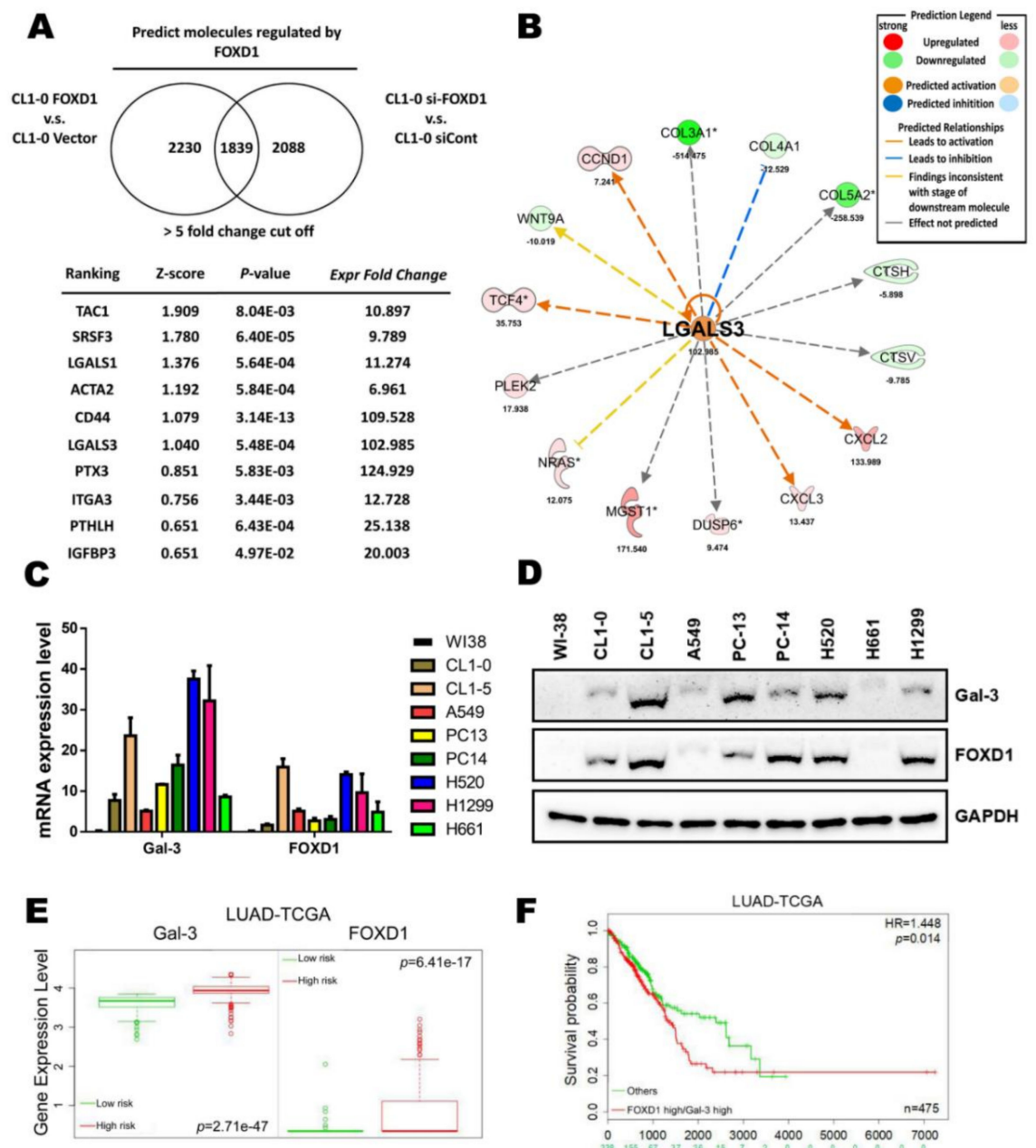

F
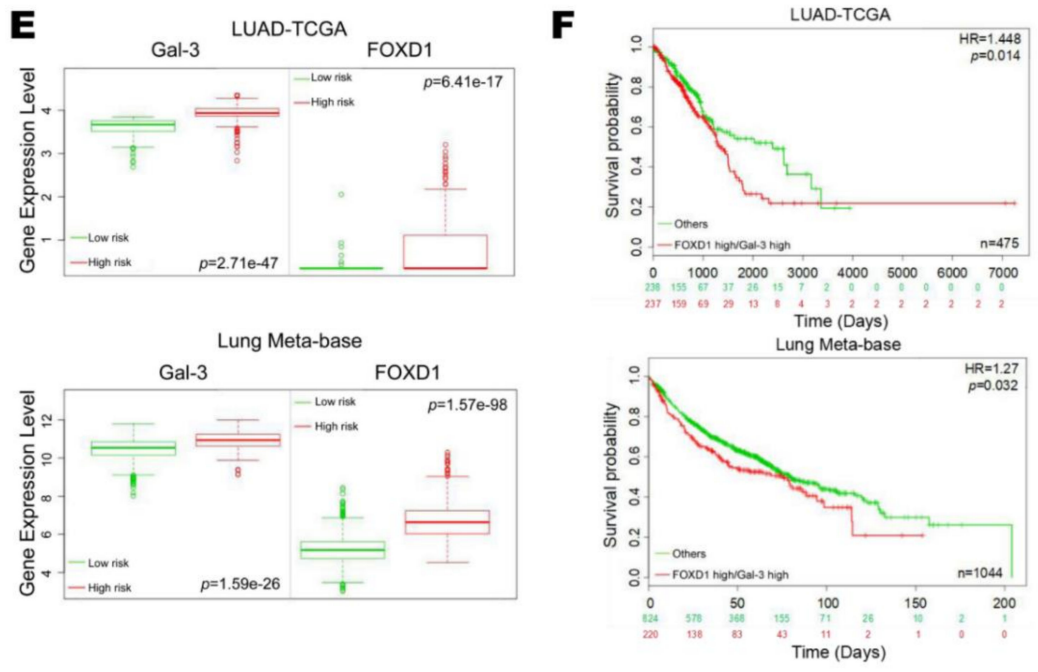

Figure 1. High FOXD1 and Gal-3 expression are associated with the poor prognosis in lung cancer. (A) The ranking of candidate molecules and the corresponding $z$-score and $p$-value using the Ingenuity Pathway Analyses (IPA) database from a microarray experiment performed with CL1-0 FOXD1 compared to CL1-0 vector and CL1-5 scramble compared to CL1-5 siRNA targeting FOXD1 (si-FOXD1) using a 5-fold change cut-off. (B) Statistical $p$-values and fold change of the expression of Gal-3 (LGALS3) and its relative molecules are shown. Prediction legend: upregulation (red round icon), downregulation (green round icon), predicted activation (brown round icon), predicted inhibition (blue round icon), leads to activation (brown line), leads to inhibition (blue line), findings inconsistent with stage of downstream molecule (yellow line), effect not predicted (gray line). (C) RT-qPCR analysis of Gal-3 and FOXD1 mRNA expression in various lung cancer cell lines. GAPDH was used as an internal control for mRNA loading. (D) Western blot analysis of Gal-3 and FOXD1 protein expression in various lung cancer cell lines. GAPDH was used as an internal control for protein loading. (E) Lung cancer patients with high gene level transcription of Gal-3 or FOXD1 expression level show a correlation with high risk and (F) poor disease-free survival. The data were retrieved and analyzed from the TCGA-LUAD samples $(n=475)$ and lung meta-base $(n=1044)$ of the SurvExpress database (http://bioinformatica.mty.itesm.mx:8080/Biomatec/SurvivaX.jsp). 


\subsection{FOXD1 is a Transcription Factor of Gal-3}

To understand whether FOXD1 upregulated Gal-3 by directly binding to the Gal-3 promoter, we searched for the transcription factor binding site in the Gal-3 promoter sequence from the JASPAR database (http://jaspar.genereg.net/). We found that Gal-3 promoter contains the FOXD1 binding sequences (TCCATAGTTTACATAAG). A luciferase promoter assay was then performed to confirm the prediction. As shown in Figure 2A, Gal-3 promoter activity was enhanced 5.8-fold by ectopic FOXD1 expression compared to without FOXD1 stimulation $(p<0.01)$. Moreover, mutation of the FOXD1 binding motif from TAGTTTAC to TAACCTGC decreased FOXD1-mediated Gal-3 promoter activity. To examine whether FOXD1 can directly bind to the Gal-3 promoter, an ChIP-qPCR assay was performed. FOXD1 was found to bind to the promoter region (-1075 to $-1058 \mathrm{nt})$ of the Gal-3 gene in human lung cancer cells (Figure 2B). To further verify whether FOXD1 could act as an upstream factor to regulate Gal-3 expression, FOXD1 was overexpressed by FOXD1 cDNA or knocked down by specific siRNA. Both qPCR and Western blot assays revealed that FOXD1 overexpression resulted in the upregulation of Gal-3 (Figure 2C); in contrast, FOXD1 depletion resulted in the downregulation of Gal-3 (Figure 2D). Furthermore, we observed that the overexpression of FOXD1 increased the proliferation and colony-forming ability of lung cancer cells, while the depletion of Gal-3 attenuated the phenotypes induced by FOXD1 (Figure 2E,F). Moreover, increased cancer cell migration and invasion ability are consistent with the FOXD1-overexpression models (Figure 2G). These results indicated that FOXD1 transactivates Gal-3 expression to promote lung cancer aggressiveness.

Recently, FOXD1 has been identified in both the cytosol and the nucleus [13]. To further determine which molecules control FOXD1 translocation into the nucleus to regulate Gal-3, we used Group-based prediction system (GPS). As FOXD1 has two ERK interaction sites (T135 and S141) in its nuclear localization signal (Figure S2A), we suspected that its translocation might be controlled by ERK1 and/or ERK2. Immunofluorescence staining revealed that both ERK and FOXD1 localized to the cytosol and the nucleus of CL1-5 cells (Figure S2B). Since ERK has been reported to activate the expression of different genes by associating with different transcription coactivators, such as Runx1 to regulate Gal-3 expression [25,30], we examined whether ERK is involved in FOXD1-mediated Gal-3 expression. Using an ERK inhibitor (PD98059) to reduce ERK phosphorylation, we showed that inhibition of the phosphorylation of ERK decreased the expression levels of FOXD1 and Gal-3 (Figure S2C). The knockdown of ERK1 or ERK2 by siRNA in CL1-5 cells suppressed FOXD1 and Gal-3 expression (Figure S2D). Moreover, the inactivation of ERK attenuated FOXD1 translocation into the nucleus (Figure S2E). Similar to the ERK inhibitor results, the depletion of ERK1 or ERK2 reduced FOXD1 in the nucleus and retained the level of FOXD1 in the cytosol (Figure S2F). To elucidate whether ERK directly interacts with FOXD1, we examined the association between ERK and FOXD1 by treating cells with the ERK inhibitor (PD98059). The immunoprecipitation assay showed that ERK interacted with FOXD1 in both the cytosol and nucleus. The downregulation of phosphorylated ERK ${ }^{\mathrm{T} 185 / \mathrm{Y} 187}$ (p-ERK) expression (but not ERK) by PD98059 suppressed the interaction of ERK and FOXD1 in the cytosol and nucleus (Figure S2G). Similar to the ERK inhibitor results, the interaction of p-ERK and FOXD1 was downregulated by the knockdown of ERK1 or ERK2 (Figure S2H). These results suggest that ERK1/2 interacts with FOXD1 in both the cytosol and nucleus, and p-ERK is required for FOXD1 translocation into nucleus.

To further determine whether ERK is directly involved in FOXD1 binding to the Gal-3 promoter, we performed a luciferase assay to measure the Gal-3 promoter luciferase activity upon the suppression of p-ERK. As shown in Figure S3A, FOXD1-mediated Gal-3 luciferase activity was markedly decreased by treating cells with the ERK inhibitor PD98059 (left) or by the depletion of ERK1 or ERK2 (right) $(p<0.001)$. Moreover, a ChIP-qPCR assay also revealed that the inactivation of ERK or the knockdown of ERK1 and ERK2 attenuated FOXD1 binding to the Gal-3 promoter (Figure S3B). Functionally, FOXD1-mediated cell proliferation was suppressed upon the inactivation of ERK signaling by inhibitors or by transfection with ERK1-siRNA or ERK2-siRNA ( $p<0.01$ ) (Figure S3C,D). In addition, the colony-formation ability promoted by FOXD1 was also suppressed by PD98059 $(p<0.001)$ or the depletion of ERK1/ERK2 
$(p<0.001)$ (Figure S3E,F). The FOXD1-mediated migration/invasion was suppressed when CL1-0 cells were treated with an ERK inhibitor or the depletion of ERK1 or ERK2 $(p<0.001)$ (Figure S3G,H). Taken together, we concluded that FOXD1 associated with p-ERK1/2 to translocate into the nucleus and bind to the Gal-3 promoter through the interaction with FOXD1.

A

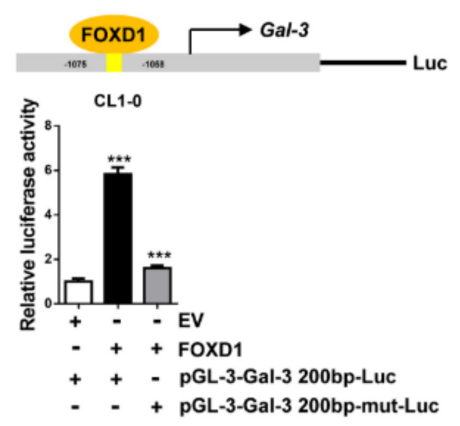

C

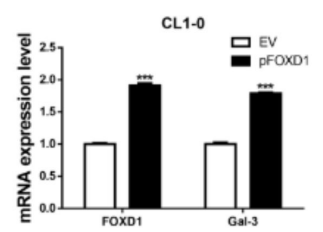

E

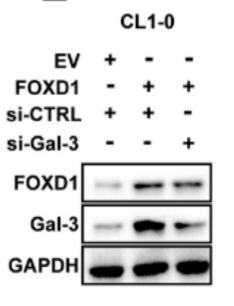

G
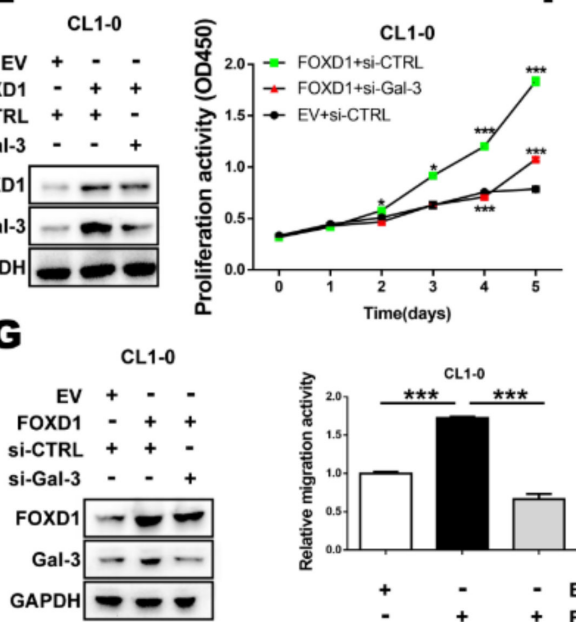

B
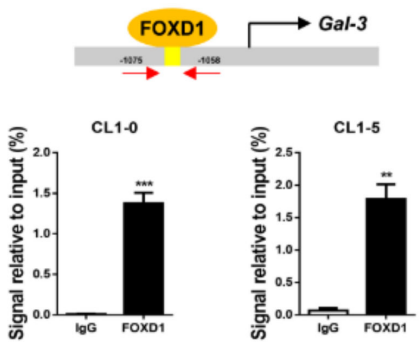

D
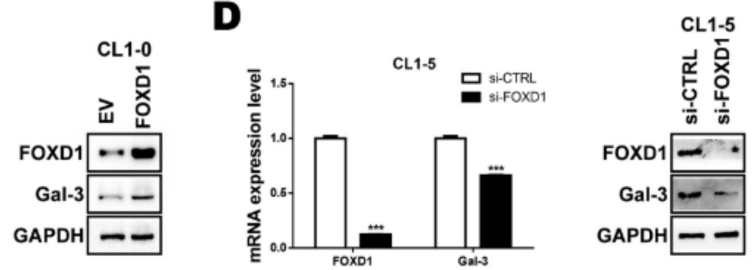

$\mathbf{F}$

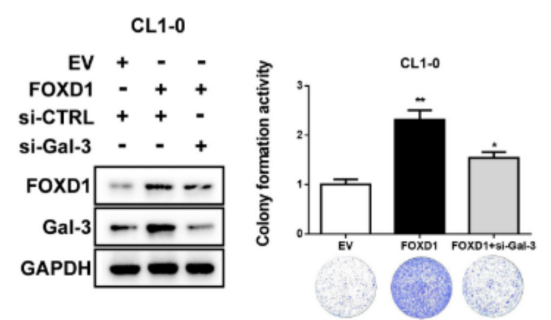

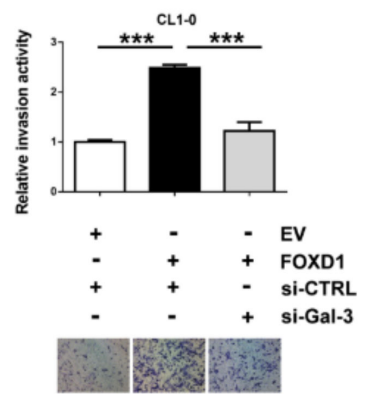

Figure 2. FOXD1 is a transcription factor of Gal-3. (A) CL1-0 cells were co-transfected with plasmids of the Gal-3 promoter reporter (pGL3-Gal-3 $2000 \mathrm{bp}$ ) or Gal-3 promoter mutation reporter (pGL3-Gal-3 2000 bp-mut) and FOXD1 (pFOXD1). The cell lysates were collected, and the promoter activity of Gal-3 was analyzed by luciferase assay. (B) ChIP-qPCR assay using IgG as a control or FOXD1 antibody in CL1-0 and CL1-5 showed the binding of FOXD1 on the Gal-3 promoter. (C) CL1-0 cells transfected with the empty vector or plasmid of FOXD1 ( $p F O X D 1$ ) for $48 \mathrm{~h}$ and (D) CL1-5 cells transfected with scramble or siRNA targeting FOXD1 (si-FOXD1) for $72 \mathrm{~h}$ were collected. The mRNA and protein levels of FOXD1 and Gal-3 were analyzed by qPCR (left) and immunoblotting (right), respectively. (E) CCK-8 assay in different groups. FOXD1-stimulated CL1-0 proliferation ability was reduced by knocking down Gal-3. (F) The colony-formation ability of FOXD1-stimulated CL1-0 cells was suppressed by the depletion of Gal-3. (G) The numbers of migrating or invading cells were increased in the FOXD1 group compared with the FOXD1-stimulated CL1-0-siGal-3 group and control group. 


\subsection{ETS-1 Is Involved in Gal-3-Mediated FOXD1 Expression}

To further identify molecules involved in Gal-3-mediated lung cancer aggressiveness, Gal-3 was overexpressed by Gal-3 cDNA or knocked down by specific siRNA. mRNA microarrays and comparative gene expression analyses were performed. Ingenuity Pathway Analyses showed that ETS-1 was one of the Gal-3 downstream effectors (Figure 3A-B). Both qPCR and Western blot assays revealed that Gal-3 depletion resulted in the downregulation of ETS-1 (Figure 3C, left). In contrast, Gal-3 overexpression resulted in the upregulation of ETS-1 (Figure 3D, right). We next investigated whether ETS-1 regulated FOXD1. We found that the depletion of ETS-1 downregulated FOXD1 at both the mRNA and protein levels in CL1-5 cells (Figure 3E-F, left), whereas the overexpression ETS-1 upregulated FOXD1 at the mRNA and protein levels in CL1-0 cells (Figure 3E-F, right). Moreover, we found that ETS-1-mediated FOXD1 expression contributed to further increase Gal-3 expression (Figure 3E-F). Reporter and ChIP-qPCR assays revealed that FOXD1 mediated the activation of Gal-3 expression in an ETS-1-dependent manner (Figure 3G-H). These findings suggest that Gal-3 increases ETS-1 expression that not only upregulates FOXD1 but also collaborates with FOXD1 to increase FOXD1-mediated activation of Gal-3 expression.

\subsection{Gal-3 Regulates FOXD1 Expression through ITGß1 Signaling}

As the interaction of Gal-3 with integrins promotes cancer progression, and integrins are one of the ERK upstream effectors [25,31], we next investigated whether FOXD1 expression is regulated by the Gal-3/ITG $\beta 1 / E R K$ axis. First, we investigated whether Gal-3 is regulated FOXD1 through ERK. Gal-3 overexpression upregulated FOXD1 at both the mRNA and protein levels in CL1-0 cells (Figure 4A, left), whereas the depletion Gal-3 by siRNA downregulated FOXD1 at the mRNA and protein levels in CL1-5 cells (Figure 4A right). We then examined whether Gal-3 associates with ITG $\beta 1$. We found that Gal-3 associated with ITG $31 /$ FAK/PI3K/AKT/PHB1/Ras/Raf1 in the lipid rafts. Gal-3 overexpression increased, whereas knockdown of Gal-3 suppressed, the interaction (Figure 4B, left) and the phosphorylation of ITG $\beta 1 /$ FAK/PI3K/AKT/PHB1/Ras/Raf1 in the lipid rafts (Figure 4B, right). To determine whether the interaction of Gal-3 and ITG $\beta 1$ is required for FOXD1 expression, we transfected CL1-0 cells with a Gal-3 expression vector or/and ITG $\beta 1$ siRNA. The overexpression of Gal-3 by an expression vector activated ITG $\beta 1$ signaling and FOXD1 expression, whereas the co-transfection of Gal-3 cDNA and ITG $\beta 1$ siRNA to block ITG $\beta 1$ signaling resulted in the downregulation of FOXD1 (Figure 4C, left). Conversely, ectopic ITG $\beta 1$ expression increased downstream signaling and upregulated FOXD1 expression. Compared with overexpression of ITG $\beta 1$ alone, the co-transfection of the ITG $\beta 1$ expression vector and Gal-3 siRNA reduced the levels of FOXD1 (Figure 4C, right). Moreover, treating cells with rhGal-3 to mimic exo-Gal-3 inducing ITG $\beta 1$ signaling also increased FOXD1 expression (Figure S4). Since ERK1/2 regulates FOXD1 translocation into the nucleus to bind to Gal-3 promoter (Figures S2 and S3), we next performed an immunoprecipitation assay and showed that the activation of ERK increased FOXD1 translocation into the nucleus. As shown in Figure 4D, the overexpression of Gal-3 or ITG $\beta 1$ increased the interaction of ERK and FOXD1 in the cytosol and nucleus. We also compared Gal-3 promoter activity when cells were transfected with Gal-3 or ITG $\beta 1$. The reporter and ChIP-qPCR assays revealed that overexpression of Gal-3 or ITG $\beta 1$ upregulated Gal-3 promoter activity (Figure S5A,B). The biological assays showed that FOXD1 depletion attenuated cell growth, as indicated by proliferation/colony formation (Figure S5C,D). 

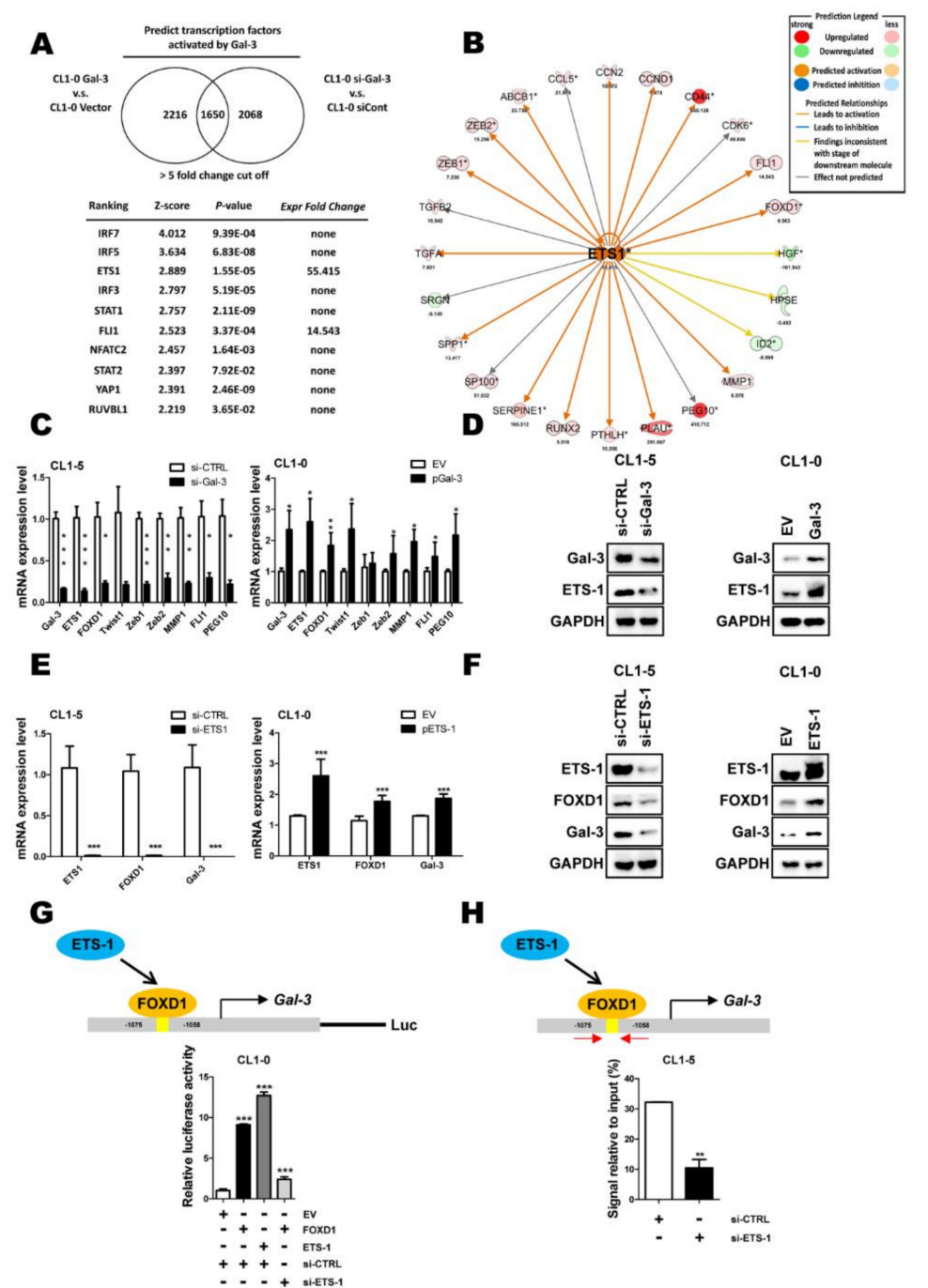

Figure 3. Proto-oncogene 1 (ETS-1) is involved in Gal-3-mediated FOXD1 expression. (A) The ranking of candidate transcription factors and its corresponding z-score and $p$-value using the IPA database from a microarray experiment performed with CL1-0 Gal-3 compared with the CL1-0 vector and CL1-5 scramble compared with CL1-5 si-Gal-3 using a 5-fold change cut-off. (B) Statistical $p$-values and fold change of the expressions of ETS-1 (ets-1) and its relative molecules are shown. Prediction legend: upregulation (red round icon), downregulation (green round icon), predicted activation (brown round icon), predicted inhibition (blue round icon), leads to activation (brown line), leads to inhibition (blue line), findings inconsistent with stage of downstream molecule (yellow line), effect not predicted (gray line). (C) CL1-0 and CL1-5 cells were transfected with specific cDNA for $48 \mathrm{~h}$ or siRNA for $72 \mathrm{~h}$; RT-qPCR analysis of ets-1, which targets downstream mRNA expression in CL1-0 cells. (D) Western blot analysis of Gal-3 and ETS-1 from CL1-0 with expression of the vector control (VC) or exogenous Gal-3 gene and CL1-5 cells with scramble and siRNA targeting Gal-3 (si-Gal-3). (E, F) CL1-0 and CL1-5 cells were transfected with the empty vector or plasmid of Gal-3 ( $p$ Ets-1) for $48 \mathrm{~h}$ or scramble and siRNA targeting ETS-1 (si-ets-1) for $72 \mathrm{~h}$, respectively, as indicated. The mRNA and protein levels of ETS-1, FOXD1, and Gal-3 were analyzed by qPCR and immunoblotting, respectively. Cells were transfected with scramble and siRNA targeting Ets-1 for $72 \mathrm{~h}$, or empty vector or plasmid of Ets-1 for $48 \mathrm{~h}$, respectively, as indicated, and immunoprecipitation was analyzed by immunoblotting. Input, the whole cell lysates. (G) CL1-0 cells were co-transfected with plasmids of Gal-3 promoter reporter (pGL3-Gal-3 2000 bp), empty vector or plasmid of FOXD1 (pFOXD1) or scramble and siRNA targeting ETS-1 (si-ets-1). Cell lysates were collected, and the promoter activity of Gal-3 was analyzed by luciferase assay. (H) CL1-5 cells were transfected with scramble and siRNA targeting ETS-1 (si-ets-1). ChIP-qPCR assay using IgG as a control or FOXD1 antibody in CL1-5 showed the binding of FOXD1 on the Gal-3 promoter. 
A
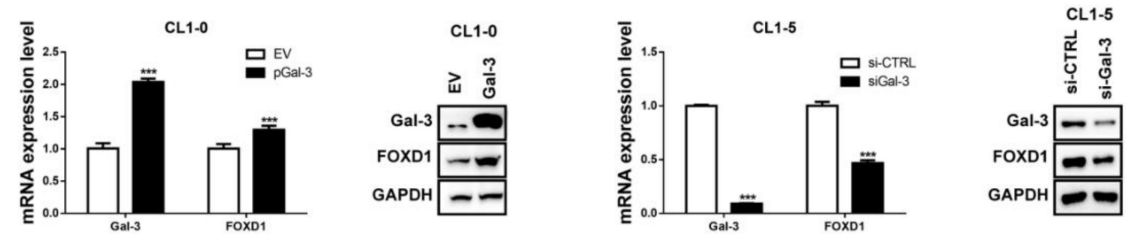

B

CL1-5

IP: IgG ITGB1

$\mathrm{EV}-++$

si-Cont - + - +

si-Cont $=+-+$

Gal-3 - -

ITGB1

FAK - - -

PI3K - -

AKT

Ras $\infty$

Raf-1 - - -

Gal-3

ITGB

FAK D-

PI3K - -

АKT $-0-0$

PHB1 200

Ras $=00$

Raf-1 - - -

Caveolin-1

Clathrin $\mathrm{HC}$

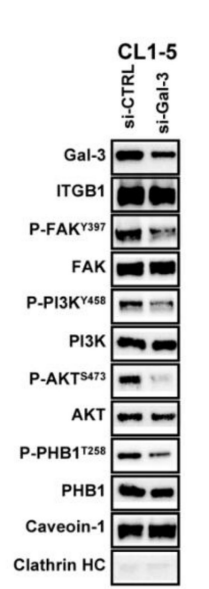

C

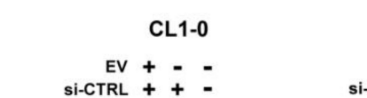

CL1-0

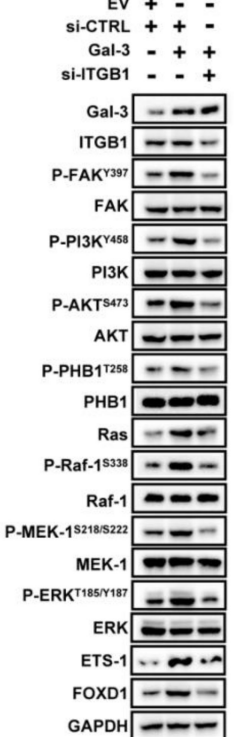

$\mathrm{EV}+-=$

ITGB1 - +

si-Gal-3 - + +

Gal-3 - -

ITGB1 - -

P-FAK ${ }^{\text {Y39 }}-\ldots$

FAK $=0$

P-PI3K ${ }^{\text {Y458 }}$

PI3K 00

P-AKT $^{\mathrm{S} 473}$

АКT - -

P-PHB17258

PHB1 1

Ras $-\ldots$

P-Raf-1 ${ }^{\mathrm{S} 338}-\infty$

Raf-1 $\infty \rightarrow \infty$

P-MEK-152181:5222

MEK-1

P-ERK ${ }^{T 185}$ 187 10

ERK 두요

ETS-1 $\cdots$

FOXD1 - - -

GAPDH

D
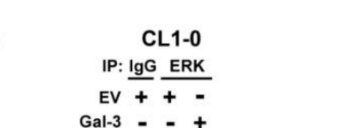

CL1-0

IP: $\operatorname{lgG}$ ERK

ITGB1 - - +
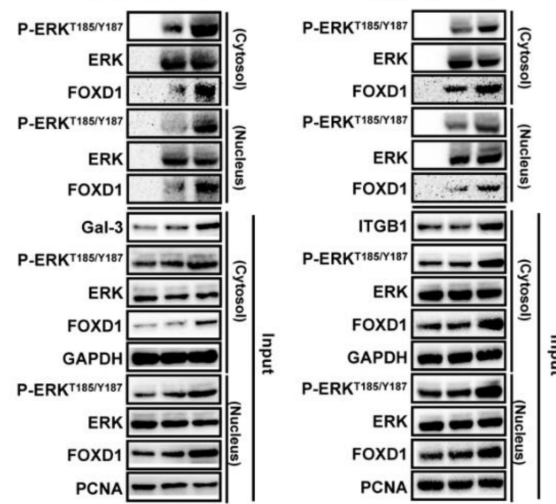

Figure 4. Gal-3 regulates FOXD1 expression through integrin- $\beta 1$ (ITG $\beta 1$ ) signaling. (A,B) CL1-0 and CL1-5 cells were transfected with the empty vector or plasmid of Gal-3 ( $p$ Gal-3) for $48 \mathrm{~h}$ or scramble and siRNA targeting Gal-3 (si-Gal-3) for $72 \mathrm{~h}$, respectively, as indicated. The mRNA and protein levels of FOXD1 and Gal-3 were analyzed by qPCR and immunoblotting. CL1-5 cells were transfected with scramble and siRNA targeting Gal-3 for 72 h or empty vector or plasmid of Gal-3 for 48 h, respectively, as indicated, and immunoprecipitation was analyzed by immunoblotting. Input, the whole cell lysates. (C) CL1-0 cells were transfected with scramble, Gal-3, ITGß1 or si-Gal-3, and si-ITGß1 for $72 \mathrm{~h}$, as indicated. Cells lysates were collected and analyzed by immunoblotting. (D) CL1-0 cells were transfected with Gal-3 or ITG $\beta 1$ expression vector for $48 \mathrm{~h}$, and immunoprecipitation was analyzed by immunoblotting.

Transwell assays showed that Gal-3-mediated cell migration/invasion ability could be attenuated by FOXD1 depletion in CL1-0 cells (Figure S5E). Taken together, these findings suggest that ITG $\beta 1 / E R K$ signaling mediates the relationship between Gal-3 and FOXD1 in lung cancer. 


\subsection{FOXD1 and Gal-3 form a Positive Regulatory Loop to Promote Lung Cancer Cell Growth and Motility}

To examine whether FOXD1 and Gal-3 may form a positive regulatory loop to promote tumor cell aggressiveness, gene manipulation of each gene was performed. Our results showed that the ectopic expression of FOXD1 or Gal-3 enhanced proliferation/colony formation and cell migration/invasion in CL1-0 cells (Figure S6A,C,E and Figure S7A,C,E). Conversely, the depletion of FOXD1 or Gal-3 decreased proliferation/colony formation and cell migration/invasion in CL1-5 cells (Figure S6B,D,F, and Figure S7B,D,F). To evaluate the feed-forward loop effects of both FOXD1 and Gal-3 on tumor cells, the cells were transfected with FOXD1 and Gal-3 siRNA. The depletion of both FOXD1 and Gal-3 resulted in the downregulation of FOXD1 and Gal-3 in an additive manner (Figure 5A). Functionally, the co-knockdown of FOXD1 and Gal-3 further attenuated cell growth and migration/invasion in CL1-5 cells (Figure 5A-C). In contrast, ectopic enforced expression of both FOXD1 and Gal-3 further increased their protein expression (Figure 5D) coupled with enhanced cell growth and migration/invasion in CL1-0 cells (Figure 5D-F). These data demonstrated that FOXD1 and Gal-3 may form a positive regulatory loop to promote lung cancer aggressiveness.
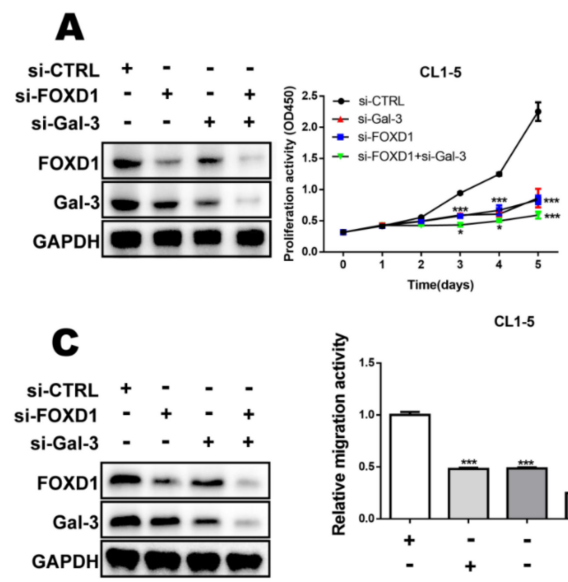

D

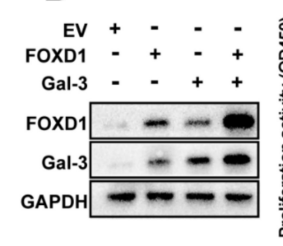

F
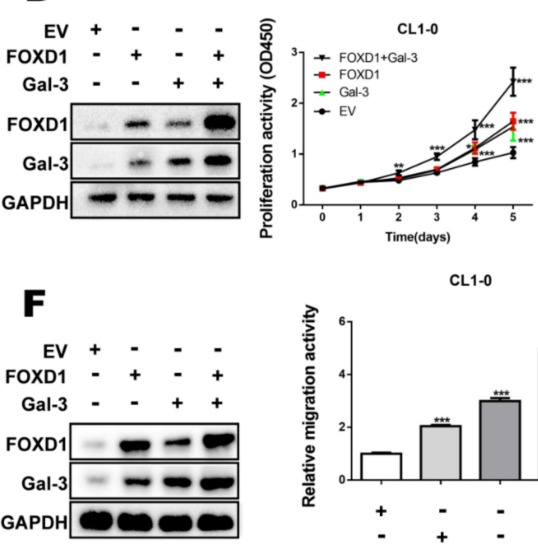

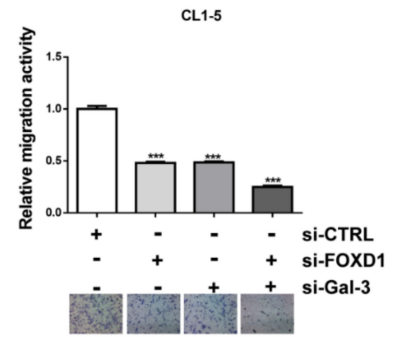

E
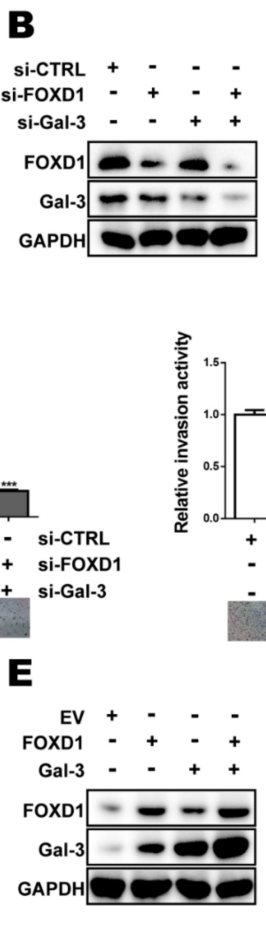

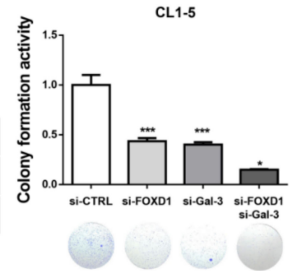

CL1-5

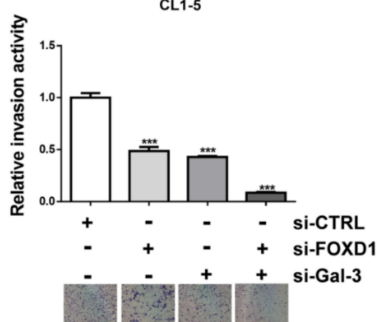

E CL1-0

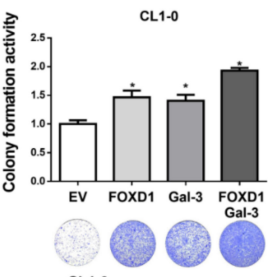

CL1-0

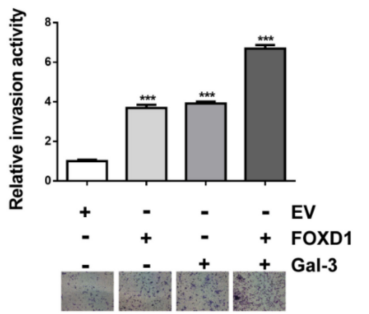

Figure 5. FOXD1 and Gal-3 form a positive regulatory loop to promote lung cancer cell growth and motility. CL1-5 cells were transfected with scramble, si-FOXD1, or si-Gal-3 or si-FOXD1 with si-Gal-3 for $72 \mathrm{~h}$. Cells lysates were collected and analyzed by immunoblotting. (A) The proliferation ability was analyzed by CCK-8. (B) Representative images of the colony-forming assay. (C) Representative images of the migration and invasion assay. CL1-0 cells were transfected with the empty vector, FOXD1, Gal-3, or both FOXD1 and Gal-3 expression vector for $48 \mathrm{~h}$. (D) Proliferation ability was analyzed by CCK-8 assay. (E) Representative images of colony-forming assay. (F) Representative images of migration and invasion assay. 


\subsection{FOXD1 and Gal-3 are Positively Correlated in Human Lung Cancer Tissues}

Accumulating evidence suggests that both FOXD1 and Gal-3 are highly expressed in several cancers $[15,16,21]$, whereas the clinicopathological relationship is still not well understood. To evaluate the correlation between FOXD1 and Gal-3 in lung cancer tissues, we determined FOXD1 or Gal-3 expression by immunochemistry staining in human lung cancer tissue microarrays. FOXD1 and Gal-3 were increased in lung tumor tissues compared with those in lung normal tissues $(p<0.001)$ (Figure 6A,B), increased in T3 + T4-size tumors $(n=23)$ compared with those in $\mathrm{T} 1+\mathrm{T} 2$ size tumors $(n=87)(p<0.001)$, increased in tumors with those in lymph node involvement $(\mathrm{N} 1+\mathrm{N} 2, n=53)$ compared with those in tumors without lymph node involvement (N0, $n=57)(p<0.001)$, and increased in stages II + III + IV $(n=56)$ compared with in stage I $(n=54)$ tumors $(p<0.001)$. Importantly, we also found that a high level of FOXD1 expression positively correlated with Gal-3 expression $(r=0.624$, $p<0.0001$, Figure S8). Overall, these data indicated that FOXD1 and Gal-3 form a positive relationship to promote tumor progression in human lung cancer tissues.
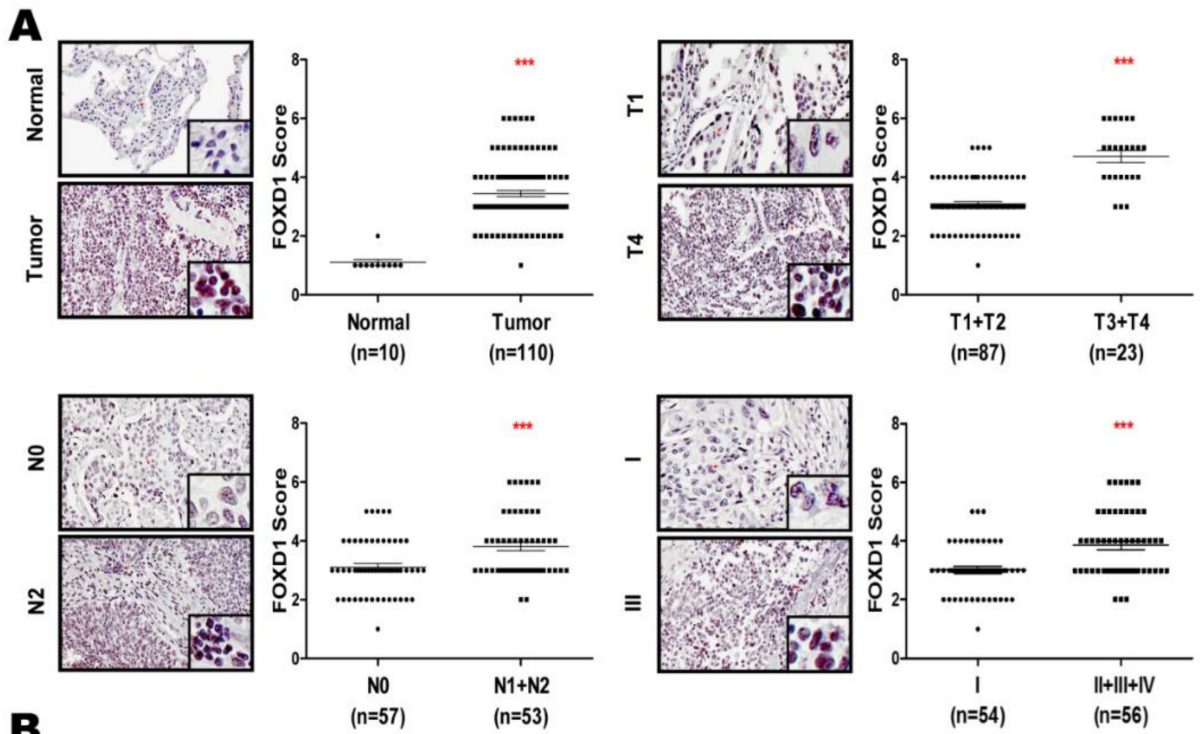

B
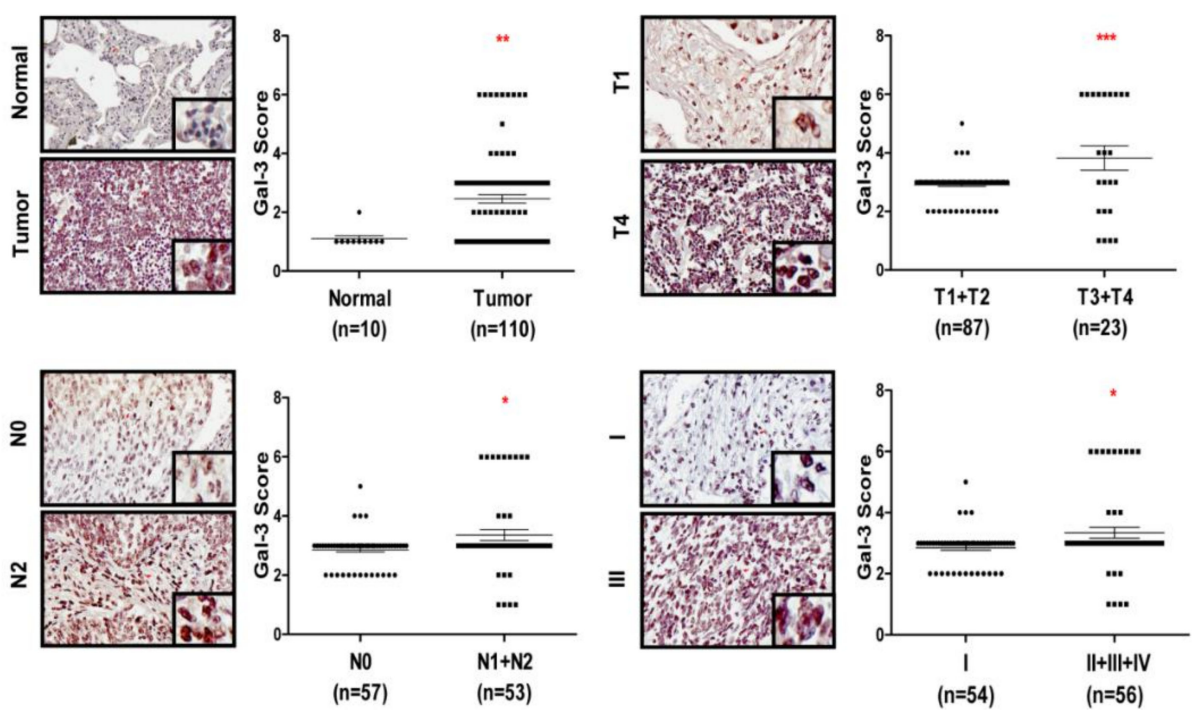

Figure 6. FOXD1 and Gal-3 are positively correlated in human lung cancer tissues. (A) Immunohistochemistry images show that FOXD1 was increased in human lung cancer tissue microarray. (B) Immunohistochemistry images show that Gal-3 was highly expressed in human lung cancer tissue microarray. 


\section{Discussion}

Tumor heterogeneity is one of the reasons for malignancy and treatment failure, including in lung cancer [32]. Insights into the regulation of cancer may provide directions for the development of new therapeutic strategies. In this study, we demonstrated that FOXD1 promoted lung cancer aggressiveness by targeting Gal-3, which acts as an oncogene in lung cancer [26] (Figure 2). Our results show that ERK1/2 regulated Gal-3 by associating with FOXD1 in both the cytosol and the nucleus. (Figures S2 and S3). In addition, Gal-3 regulated FOXD1 expression through the ITG $\beta 1 / E R K / E T S-1$ cascade (Figure 4). FOXD1 and Gal-3 form a positive loop that promotes lung cancer aggressiveness (Figure 5).

The dysregulation of FOXD1 expression has previously been associated with malignant behavior in certain cancers and has been reported to contribute to aspects of tumor progression, such as drug resistance, metastasis, and stemness [11]. Aberrant FOXD1 mRNA expression was recognized as an independent marker in NSCLC patients [15]. Consistently, we and others have shown that elevated FOXD1 protein expression can be detected in clinical lung cancer tissues and associated with tumor malignancy (Figure 6) [16]. Ectopic or depleted expression further suggested that FOXD1 promotes the growth and motility of lung cancer cells (Figure S6). FOXD1 expression was also related to the expression of different EMT markers (Figure S6). These results are consistent with those of a previous study in which FOXD1 promoted cell proliferation and motility through vimentin activation in NSCLC [16]. These data, together with the previous report, reveal that targeting FOXD1 may improve interventions in lung cancer. It is well documented that FOXD1 contributes to aggressiveness by targeting different downstream molecules in various tumor types [11]. The tumorigenic role of FOXD1 has been identified; however, the oncogenic role remains controversial in various cancers. For example, in medulloblastoma, FOXD1 acts as a tumor suppressor by targeting NKX2.2. In contrast, in breast cancer, FOXD1 promotes drug resistance by downregulating P27 [12]. In glioma, FOXD1 upregulates ALDH1A3 to promote stemness properties [13]. FOXD1 also targets RAC1B to regulate melanoma cell motility [17]. However, the downstream target involved in FOXD1 in lung cancer remains to be characterized. Here, we provide evidence that links FOXD1 with Gal-3 in lung cancer, in which FOXD1 enhanced Gal-3 expression (Figure 2); subsequently, Gal-3-mediated ITG $\beta 1 /$ ETS-1 signaling contributed to further increase FOXD1 expression (Figure 4). In particular, we identified this feedback loop that likely sustains the aggressiveness of lung cancers.

Gal-3 belongs to chimera type of $\beta$-galactoside-binding lectin [18]. Accumulating evidence demonstrates that in lung cancer, elevated Gal-3 is linked to the promotion of tumor progression [21,29]. Consistent with this model, gain- and loss-of function experiments were used to evaluate the promotion of growth and motility of lung cancer cells by Gal-3 (Figure S7). We and others have suggested that Gal-3 expression correlated with lymphatic metastasis and tumor progression in lung cancer patients (Figure 6) [21]. The transcription factor Runx1 has been identified as being involved in Gal-3 expression [30]. However, a previous study reported that the knockdown of RUNX1 increased lung cancer cell growth and motility, and lung cancer patients with low RUNX1 exhibit a poor prognosis [28]. Intriguingly, there is no report of FOXD1 being associated with Gal-3 expression. Notably, we identified that Gal-3 has a consensus binding sequence of FOXD1 (TAGTTTAC) on the Gal-3 promoter (-1075 to $-1058 \mathrm{nt}$ ), which has ability to recruit the FOXD1 interaction (Figure 2). Our data demonstrate that Gal-3 is transcriptionally regulated by FOXD1 (Figure 2B), thereby promoting metastasis ability (Figure 2G).

More recent studies suggested that FOXD1 localizes to the cytosol and nucleus [13]. However, the mechanism of the regulatory action of FOXD1 translocation has not been determined. Group-based prediction system (GPS) prediction shows that FOXD1 has an ERK association site in nuclear localization signals (NLS). ERK is known to activate the expression of different genes by association with different transcriptional co-activators $[30,33,34]$. In addition, several reports have shown that ERK signaling acts through association with Runx1 to regulate Gal-3 [25,30]. To our knowledge, this report is the first to show ERK1/2 interaction with FOXD1 in both the cytosol and nucleus (Figure S2), in which the 
inactivation of ERK or the depletion ERK1 or ERK2 suppresses the translocation of FOXD1 into the nucleus to transactivate Gal-3 expression (Figure S3). In response to signal transduction, the catalytic domain of the ERK1/2 kinase activity requires dual phosphorylation, and using an inhibitor is not sufficient to discriminate which form of ERK is critical for FOXD1 translocation [35]. Our data suggest that both ERK1 and ERK2 were essential for FOXD1 translocation (Figures S2 and S3); therefore, FOXD1-mediated cell growth and motility were regulated by ERK signaling (Figure S3). These results indicate that ERK activation increases the interaction of ERK with FOXD1 in the cytosol, leading to translocation into the nucleus to bind the Gal-3 promoter and to promote tumor growth and motility. In this study, we found that FOXD1 expression or translocation was regulated by ERK (Figures S2 and S3). Notably, a previous study reported that depletion of Gal-3 led to ERK dephosphorylation in PDAC cells [36]. In this study, for the first time, we found that FOXD1 could also be regulated by Gal-3 (Figures 3 and 4); however, the underlying mechanism involved in Gal-3-mediated FOXD1 was not fully elucidated. In this study, we found that FOXD1 expression was regulated by ETS-1 (Figure 3). As Gal-3 can phosphorylate ERK signaling through the association with different molecules on the cell surface, including ITG $\beta 1[25,29]$, it is likely that ERK-mediated FOXD1 translocation can, at least partially, be regulated by the Gal-3-mediated ITG $\beta 1 / E R K / E T S-1$ cascade. The overexpressing and/or knockdown of

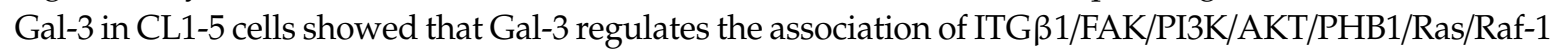
in lipid rafts (Figure 4); this result is also consistent with our previous report [37]. Our findings validated Gal-3-enhanced FOXD1 expression as being dependent on the presence of ITG $\beta 1$ (Figure $4 \mathrm{C}$ ). In addition, the upregulation of Gal-3 activates ERK and promotes the interaction of FOXD1 and ERK in the cytosol and nucleus (Figure 4D), which in turn activates Gal-3 expression (Figure S5A,B). Therefore, Gal-3 stimulated proliferation, colony formation, migration, and invasion, and these effects were attenuated by downregulating FOXD1 (Figure S5C-E). These results indicate that ITG $\beta 1 /$ ERK signaling bridges the relationship between Gal-3 and FOXD1, and FOXD1 and Gal-3 form a positive feedback loop in lung cancer. However, we cannot rule out the possibility that Gal-3 may regulate FOXD1 through a different molecule on the cell surface.

Given the relationship between FOXD1 and Gal-3, it is most likely that targeting both FOXD1 and Gal-3 may have greater effects on tumor progression that targeting either protein alone [11,38]. In support of this possibility, co-knockdown not only resulted in a reduction of FOXD1 and Gal-3 expression but also further suppressed tumor growth and motility (Figure 5A-C). Conversely, co-upregulation resulted in the increase in the expression of these proteins (Figure 5D-F). Our findings demonstrate that this positive feedback loop between FOXD1 and Gal-3 might regulate lung cancer aggressiveness in an additive manner.

Abnormal expression of FOXD1 and Gal-3 is known to contribute to cancer pathogenesis [15,16,21], which makes them therapeutic targets for various cancers including lung cancer. However, the precise clinical pathological correlation between FOXD1 and Gal-3 remains poorly defined. In this study, we evaluated the protein levels of FOXD1 and Gal-3 in clinical human lung tumor microarrays. We and others have shown that FOXD1 or Gal-3 are increased in advanced tumor tissues (Figure 6A-B) $[15,16,21]$. Based on the score of the tissues, we provided new evidence that the FOXD1 expression level positively correlated with Gal-3 in human lung tissues (Figure S8). These data led us to hypothesize that FOXD1 and Gal-3 form a regulatory circuit during the tumorigenesis of lung cancer. A schematic diagram is presented to illustrate the interrelationships between FOXD1 and Gal-3 (Figure 7), 


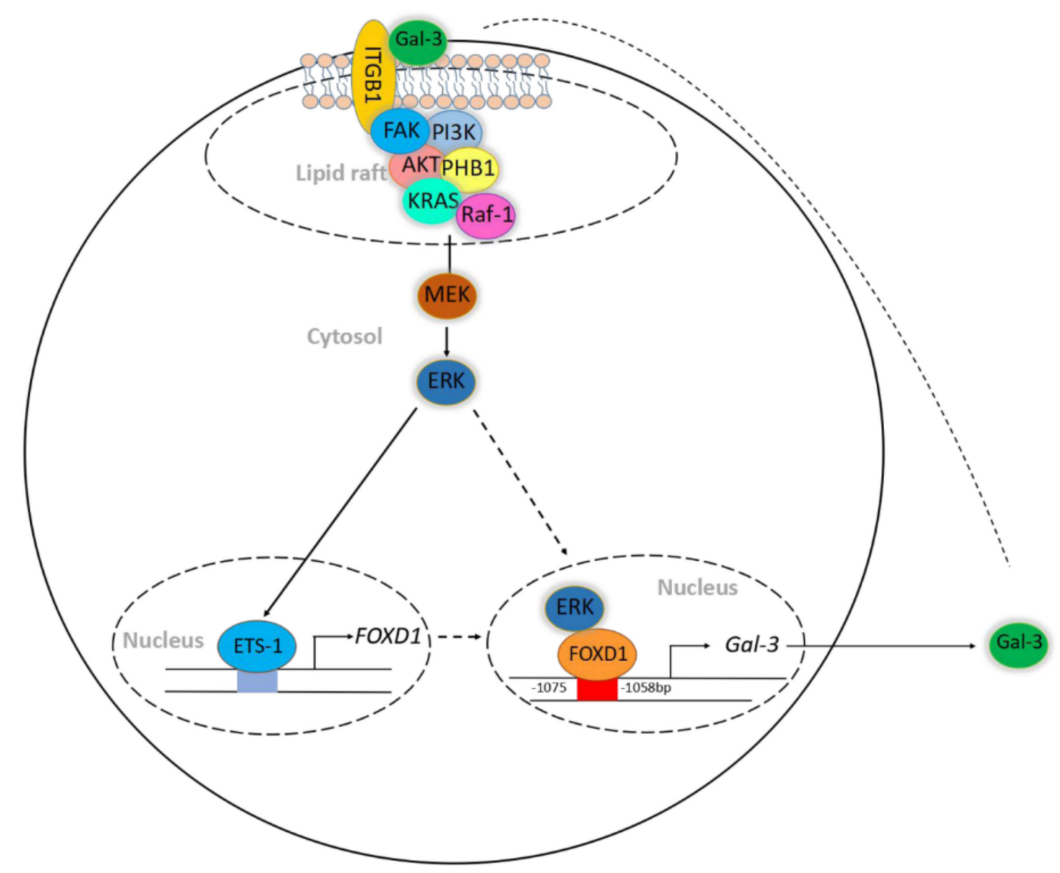

Figure 7. Schematic diagram illustrates cooperation between FOXD1 and Gal-3 in promoting lung cancer proliferation and metastasis.

\section{Materials and Methods}

\subsection{Cell Culture}

Lung cell lines were a kind gift from Michael Hsiao (Academia Sinica, Taiwan) and lung cancer cells were maintained in RPMI1640 medium (Gibco, USA) (CL1-0, CL1-5, H1299, H520, H661) or Dulbecco's modified Eagle medium (DMEM) (Gibco, USA) (A549, PC13, PC14) and Minimum Essential Eagle Medium with Earle's BSS (MEM Eagle EBSS) (Gibco, USA) (WI-38) containing 10\% fetal bovine serum (FBS; Gibco, USA), supplemented with 100 units $/ \mathrm{mL}$ penicillin and $100 \mu \mathrm{g} / \mathrm{ml}$ streptomycin. Cells were maintained in a $37^{\circ} \mathrm{C}$ incubator with a humidified atmosphere containing $5 \% \mathrm{CO}_{2}$.

\subsection{Transfection}

Cells were used from passages three to six. GenMute transfection reagent (SignaGen Laboratories, MD, USA) were used to transfect various siRNA and plasmids.

\section{3. mRNA-Based qPCR Assay}

Cell RNA was extracted using RNA extraction kit according to the manufacturer's protocol (Invitrogen, USA). cDNA was reversed by ToolsQuant II Fast RT kit (Tools Biotech, Taiwan). The mRNA exchange fold was quantitated by the real-time RT-PCR using the Fast SYBR Green Master Mix (QIAGEN, Germany). The PCR protocol was followed by 7500 Fast Real-Time PCR System (Thermo Scientific, USA). The relative expression of the genes was normalized by GAPDH as an internal control.

\subsection{Immunoblotting and Immunoprecipitation}

Harvested cells were lysed by RIPA lysis buffer (Santa Cruz Biotech, USA) containing protease and phosphatase inhibitors, and the proteins were loaded in equal amounts into optimal sodium dodecyl sulfate-polyacrylamide gel electrophoresis (SDS-PAGE). Followed by gel electrophoresis, the separated proteins were transferred to the 0.45- $\mu \mathrm{m}$ PVDF membrane (GE Healthcare Amersham, UK). The membranes were incubated with $5 \%$ skim milk (Difco, BD, USA) to block the non-specific signal, then incubated with specific primary antibodies at $4{ }^{\circ} \mathrm{C}$ overnight. On the next day, the membrane 
was incubated with horseradish peroxidase (HRP)-conjugated secondary antibody at room temperature for $1 \mathrm{~h}$. Peroxidase activity was detected using chemiluminescence (Perkim Elmer, USA), and the intensity was quantified by software ImagePro. For immunoprecipitation, harvested cells were immunoprecipitated with specific primary antibody or control IgG, together with protein G Mag Sepharose overnight. Collection of the immunoprecipitated pellet was done with MagRack6, and the pellet was washed with RIPA buffer, non-immunoprecipitated lysate (Input) and immunoprecipitated lysate were immunoblotted with the indicated antibody.

\subsection{Immunohistochemistry Analysis}

Clinical lung carcinoma tissue microarray slides (BC041115d, US Biomax, Germany). The slide was de-paraffinized with xylene, rehydrated with alcohol and antigen retrieval, and then blocked with 5\% normal goat serum for one hour at room temperature and then incubated with the FOXD1 antibody (Aviva Systems Biology, OAAB10686, USA) or Gal-3 antibody (R\&D, MAB11541, USA) at $4{ }^{\circ} \mathrm{C}$ overnight. The next day, after washing with phosphate-buffered saline (PBS) the slides were incubated with goat anti-rabbit Fab at room temperature for $30 \mathrm{~min}$, and labelled with $\mathrm{ABC}$ reagent. Then, the slides were further stained with DAB reagent. Stained slides were scanned by a ScanscopeXT system (Aperio Technologies, Vista, CA, USA) and positively stained cells in 10 fields of each section were analyzed by ImageScope 9.1 software (Aperio Technologies, USA). To evaluate the positive FOXD1 or Gal-3 intensity in each tissue sample, the stained level was further scored as: 0 (no staining), 1 (light brown), 2 (medium brown), or 3 (dark brown) by the percentage of positive cells, and the expression results were validated by qualified pathologist.

\subsection{Colonogenic Formation Assay}

Transfected lung cancer cells $\left(2 \times 10^{3}\right.$ cells/well) were growth in six-well plates. After 14 days, cells were fixed by methanol-acetic acid (7:1) for $30 \mathrm{mins}$, then visualized by $0.1 \%$ crystal violet (Sigma-Aldrich, USA), and counted using a stereomicroscope.

\subsection{Extraction of Cytoplasma/Nucleus and Membrane Raft Proteins}

Cells were transfected with or without specific cDNA or si-RNA for 48-72 $\mathrm{h}$, and harvested in a RIPA lysis buffer (Santa Cruz Biotech, USA) supplemented with phosphatase and protease inhibitors. Cytoplasma/nucleus and membrane raft were obtained according to the cytoplasma/nucleus and plasma membrane protein extraction kit manufacturer's instruction. (BioVision, Mountain View, CA, USA). GAPDH was used as a cytoplasma marker. Proliferating cell nuclear antigen (PCNA) was used as a nucleus marker. Caveolin-1 was used as a membrane raft fraction marker. Clathrin heavy chain (HC) was used as a non-raft fraction marker.

\subsection{Chromatin Immunoprecipitation Assay}

The CHIP-qPCR assay was followed by the EZ-Magna ChIP A/G kit instruction (EZ-Magna ChIP A/G kit; Millipore, Billerica, MA). Harvested cells were cross-linked by using $1 \%$ formaldehyde (Sigma-Aldrich, USA) to fix protein and DNA complexes. Cells were fragmented by sonication using Ultrasonic Disruptor UD-201 (TOMY, Japan) resulting in DNA fragments from 200 to $1000 \mathrm{bp}$ and DNA samples were confirmed by electrophoresis. DNA fragments containing Protein A/G magnetic beads were immunoprecipitation by anti-FOXD1 (Pierce Thermo scientific, PA5-27142) or using IgG isotype as a control antibodies in $4{ }^{\circ} \mathrm{C}$ overnight. Using the magnetic separator to isolate the DNA, the isolated DNA was analyzed by qPCR with specific Human LGALS3 primer (GPH1003937(-)10A; +333 bp from the transcription start site) (QIAGEN, Germany). 


\subsection{Promoter Assay}

The Gal-3 promoter sequence (2000 bp) and its mutation sequence were synthesized by Biotools (Biotools, Taiwan) and cloned into pGL3 luciferase reporter vector (Promega, Madison, WI, USA). For the luciferase reporter assay, optimal numbers of lung cancer cells were transfected with specific target gene plasmid and pRL-TK plasmid (Promega, USA) as an internal control for $48 \mathrm{~h}$. The luciferase activities were measured with Dual-Luciferase Reporter Assay System (Promega, USA) using a microplate reader (BioTek EL×800, USA).

\subsection{Proliferation Assay}

Transfected lung cancer cells $\left(1 \times 10^{4}\right.$ cells/well) were re-plated in a 96-well plates. We discarded the supernatant, and CCK-8 solution (Dojindo, Japan) was added to each well followed by incubation for $1 \mathrm{~h}$. Eventually, the absorbance of formazan crystals colored solution was measured at $450 \mathrm{~nm}$ by a spectrophotometer (BioTek EL×800, USA).

\subsection{Transwell Migration and Invasion Assay}

Optimal numbers of CL1-0 and CL1-5 suspended in 50-100 $\mu$ L of serum-free medium were seeded into the upper chamber with a $8 \mu \mathrm{m}$ porosity polycarbonate membrane inserts (Corning, USA), which were precoated with fibronectin $(20 \mathrm{ng} / \mathrm{mL}$; Millipore, Germany) for migration assays or with matrix gel (1 mg/mL; BD Bioscience, USA) for invasion assays. The lower chamber was contained with complete growth medium supplemented with $10 \%$ FBS as the chemoattractant. During the 16-h incubation, cells migrated through the membrane from the upper to the lower side, followed by staining with Liu's stain A and B reagent (TonYar Biotech, R.O.C.) and counted under a microscope at 200x magnification. Experiments were performed in triplicate, and three separate experiments were conducted for each group.

\subsection{Immunofluorescence Staining}

A total of $1 \times 10^{4}$ cells were seeded and grown in 5-mm glass slides for $24 \mathrm{~h}$ and subsequently fixed with methanol for $1 \mathrm{~h}$ at room temperature. The fixed cells were washed with PBS buffer and stained with a specific primary antibody for $24 \mathrm{~h}$. To distinguish the different primary antibodies, secondary antibodies were label with fluorescence (green and red) for $2 \mathrm{~h}$ at room temperature. The nuclei were staining with DAPI. The images were visualized by confocal microscopy (Zeiss LSM 780 + ELYRA, Carl Zeiss, Jena, Germany) and analyzed using ZEN software.

\subsection{In-Silicon mRNA Microarrays}

The cells were transfected with a specific cDNA vector or siRNA. RNA was extracted as follows using an RNA extraction kit. An mRNA microarray was performed by Genomics Research Center (GRC) core facility (Academia sinica, Taiwan). Transcriptional profiling data were collected from the GEO database (GSE7670) and from microarray analysis of cells with FOXD1/Gal-3 overexpression or FOXD1/Gal-3 knockdown. Then, the expression level of genes was further subjected to GeneSpring GX11 software (Agilent Technologies, Palo Alto, CA, USA) to normalize and generate log2 values. The genes that exhibited a more than 5-fold change were analyzed by Ingenuity Pathway Analysis software (IPA; QIAGEN, Valencia, CA, USA) to identify the upstream regulators and kinases that were responsive to FOXD1/Gal-3 overexpression or knockdown.

\subsection{Statistical Analysis}

All statistical comparisons were made using the two-tailed Student's $t$-test. Tissue slides were scanned and analyzed using ImageScope 9.1 software (Aperio Technologies, USA). Statistical significance was evaluated by the $t$ test at ${ }^{*}, p<0.05 ; p<0.01 ;{ }^{* *}, p<0.001$ to represent differences between groups. 


\section{Conclusions}

In summary, this study reports a novel regulatory loop between FOXD1 and Gal-3. FOXD1 and Gal-3 positively correlated with aggressiveness in human lung cancer. FOXD1 acted as a transcription factor and directly bound the promoter region of Gal-3, and FOXD1 translocation was regulated by ERK signaling. In turn, Gal-3 regulated FOXD1 activity through the ITG $\beta 1 /$ ERK/ETS-1 pathway. More importantly, we showed that the positive regulatory loop of activation of FOXD1 and Gal-3 increased the growth and motility of lung cancer. Moreover, FOXD1 combined with Gal-3 served as an independent prognostic factor for lung cancer patients. Altogether, our results provide new insights for developing a new therapeutic strategy by targeting the FOXD1/Gal-3 axis.

Supplementary Materials: The following are available online at http://www.mdpi.com/2072-6694/11/12/1897/s1. Figure S1: Relative mRNA and protein expression of FOXD1 and Gal-3 in lung cancer cell lines, Figure S2: ERK1 and ERK2 bind FOXD1 and translocate into the nucleus, Figure S3: Interaction of ERK and FOXD1 is required for Gal-3 transactivation, Figure S4: rhGal-3 regulates ETS-1/FOXD1 through ITG $\beta 1 / E R K$ signaling, Figure S5: FOXD1 is required for Gal-3-mediated cell growth and motility, Figure S6: FOXD1 promotes growth and motility of lung cancer cells, Figure S7: Gal-3 promotes growth and motility of lung cancer cells, Figure S8: Correlation between FOXD1 and Gal-3 expression. FOXD1 expression is positively related to Gal-3 expression $(r=0.624)$.

Author Contributions: Conceptualization, M.H., S.-M.L.; Data curation, C.-H.L., Y.-C.C.; Formal analysis, C.-H.L., Y.-C.C.; Funding acquisition, M.H., S.-M.L; Investigation, C.-H.L., Y.-C.C., M.H., S.-M.L.; Methodology, C.-H.L., Y.-C.C.; Project administration, M.H., S.-M.L.; Resources, M.H., S.-M.L.; Software, M.H.; Supervision, Y.-C.C., M.H., S.-M.L.; Validation, C.-H.L., Y.-C.C., S.-M.L.; Writing—original draft, C.-H.L.; Writing—review and editing, Y.-C.C., M.H., S.-M.L.

Funding: For the project was provided by the Agricultural Biotechnology Research Center (Academia Sinica, Taiwan) and the Ministry of Science and Technology (MOST 105-2320-B-001-024) to S.M.L. This research was also supported by Academia Sinica (AS-SUMMIT-108) to M.H.

Acknowledgments: We would like to thank Genomics Research Center Instrument Facilities (Academia Sinica, Taiwan) for providing the digital pathology analyses. We thanks Tracy Tsai for her great helps in immunohistochemistry works.

Conflicts of Interest: The authors declare no conflict of interest, as this study was not supported by any private company.

$\begin{array}{ll}\text { Abbreviations } & \\ \text { ETS-1: } & \text { Proto-oncogene 1 } \\ \text { FOXD1: } & \text { Forkhead box D1 } \\ \text { FOX: } & \text { Forkhead box } \\ \text { Gal-3: } & \text { Galectin-3/LGALS3 } \\ \text { GPS: } & \text { Group-based prediction system } \\ \text { HR: } & \text { Hazard ratio } \\ \text { ITG } \beta 1: & \text { Integrin- } \beta 1 \\ \text { IPA: } & \text { Ingenuity Pathway Analysis } \\ \text { NSCLC: } & \text { Non-small cell lung cancer } \\ \text { NSL: } & \text { Nuclear localization signals } \\ \text { OS: } & \text { Overall survival } \\ \text { PFS: } & \text { Progression-free survival } \\ \text { PRS: } & \text { Post-recurrence survival rate } \\ \text { TF: } & \text { Transcription factor }\end{array}$

\section{References}

1. Siegel, R.L.; Miller, K.D.; Jemal, A. Cancer statistics, 2019. CA Cancer J. Clin. 2019, 69, 7-34. [CrossRef]

2. Sun, S.; Schiller, J.H.; Spinola, M.; Minna, J.D. New molecularly targeted therapies for lung cancer. J. Clin. Investig. 2007, 117, 2740-2750. [CrossRef]

3. Simeone, J.C.; Nordstrom, B.L.; Patel, K.; Mann, H.; Klein, A.B.; Horne, L. Treatment patterns and overall survival in metastatic urothelial carcinoma in a real-world, US setting. Cancer Epidemiol. 2019, 60, 121-127. [CrossRef] 
4. Sekihara, K.; Hishida, T.; Yoshida, J.; Oki, T.; Omori, T.; Katsumata, S.; Ueda, T.; Miyoshi, T.; Goto, M.; Nakasone, S.; et al. Long-term survival outcome after postoperative recurrence of non-small-cell lung cancer: Who is 'cured' from postoperative recurrence? Eur. J. Cardio Thorac. 2017, 52, 522-528. [CrossRef]

5. Taniguchi, Y.; Tamiya, A.; Isa, S.I.; Nakahama, K.; Okishio, K.; Shiroyama, T.; Suzuki, H.; Inoue, T.; Tamiya, M.; Hirashima, T.; et al. Predictive Factors for Poor Progression-free Survival in Patients with Non-small Cell Lung Cancer Treated with Nivolumab. Anticancer Res. 2017, 37, 5857-5862. [CrossRef] [PubMed]

6. Lambert, M.; Jambon, S.; Depauw, S.; David-Cordonnier, M.H. Targeting Transcription Factors for Cancer Treatment. Molecules 2018, 23, 1479. [CrossRef] [PubMed]

7. Levinson, R.S.; Batourina, E.; Choi, C.; Vorontchikhina, M.; Kitajewski, J.; Mendelsohn, C.L. Foxd1-dependent signals control cellularity in the renal capsule, a structure required for normal renal development. Development 2005, 132, 529-539. [CrossRef] [PubMed]

8. Koga, M.; Matsuda, M.; Kawamura, T.; Sogo, T.; Shigeno, A.; Nishida, E.; Ebisuya, M. Foxd1 is a mediator and indicator of the cell reprogramming process. Nat. Commun. 2014, 5. [CrossRef] [PubMed]

9. Lin, E.E.; Sequeira-Lopez, M.L.; Gomez, R.A. RBP-J in FOXD1+ renal stromal progenitors is crucial for the proper development and assembly of the kidney vasculature and glomerular mesangial cells. Am. J. Physiol. Ren. Physiol. 2014, 306, F249-F258. [CrossRef] [PubMed]

10. Song, R.; Lopez, M.; Yosypiv, I.V. Foxd1 is an upstream regulator of the renin-angiotensin system during metanephric kidney development. Pediatr Res. 2017, 82, 855-862. [CrossRef] [PubMed]

11. Quintero-Ronderos, P.; Laissue, P. The multisystemic functions of FOXD1 in development and disease. J. Mol. Med. 2018, 96, 725-739. [CrossRef] [PubMed]

12. Zhao, Y.F.; Zhao, J.Y.; Yue, H.; Hu, K.S.; Shen, H.; Guo, Z.G.; Su, X.J. FOXD1 promotes breast cancer proliferation and chemotherapeutic drug resistance by targeting p27. Biochem. Biophys. Res. Commun. 2015, 456, 232-237. [CrossRef] [PubMed]

13. Cheng, P.; Wang, J.; Waghmare, I.; Sartini, S.; Coviello, V.; Zhang, Z.; Kim, S.H.; Mohyeldin, A.; Pavlyukov, M.S.; Minata, M.; et al. FOXD1-ALDH1A3 Signaling Is a Determinant for the Self-Renewal and Tumorigenicity of Mesenchymal Glioma Stem Cells. Cancer Res. 2016, 76, 7219-7230. [CrossRef] [PubMed]

14. Zhang, Y.M.; Wang, T.; Wang, S.; Xiong, Y.L.; Zhang, R.; Zhang, X.; Zhao, J.; Yang, A.G.; Wang, L.; Jia, L.T. Nkx2-2as Suppression Contributes to the Pathogenesis of Sonic Hedgehog Medulloblastoma. Cancer Res. 2018, 78, 962-973. [CrossRef] [PubMed]

15. Nakayama, S.; Soejima, K.; Yasuda, H.; Yoda, S.; Satomi, R.; Ikemura, S.; Terai, H.; Sato, T.; Yamaguchi, N.; Hamamoto, J.; et al. FOXD1 Expression Is Associated with Poor Prognosis in Non-small Cell Lung Cancer. Anticancer Res. 2015, 35, 261-268.

16. Li, D.; Fan, S.; Yu, F.; Zhu, X.; Song, Y.; Ye, M.; Fan, L.; Lv, Z. FOXD1 Promotes Cell Growth and Metastasis by Activation of Vimentin in NSCLC. Cell Physiol. Biochem. 2018, 51, 2716-2731. [CrossRef]

17. Wu, H.Z.; Larribere, L.; Sun, Q.; Novak, D.; Sachindra, S.; Granados, K.; Umansky, V.; Utikal, J. Loss of neural crest-associated gene FOXD1 impairs melanoma invasion and migration via RAC1B downregulation. Int. J. Cancer 2018, 143, 2962-2972. [CrossRef]

18. Hughes, R.C. Secretion of the galectin family of mammalian carbohydrate-binding proteins. Biochim. Biophys. Acta Gen. Subj. 1999, 1473, 172-185. [CrossRef]

19. Ahmed, H.; AlSadek, D.M.M. Galectin-3 as a Potential Target to Prevent Cancer Metastasis. Clin. Med. Insights Oncol. 2015, 9, 113-121. [CrossRef]

20. Cardoso, A.C.F.; Andrade, L.N.D.; Bustos, S.O.; Chammas, R. Galectin-3 Determines Tumor Cell Adaptive Strategies in Stressed Tumor Microenvironments. Front. Oncol. 2016, 6, 127. [CrossRef]

21. Chung, L.Y.; Tang, S.J.; Wu, Y.C.; Sun, G.H.; Liu, H.Y.; Sun, K.H. Galectin-3 augments tumor initiating property and tumorigenicity of lung cancer through interaction with beta-catenin. Oncotarget 2015, 6, 4936-4952. [CrossRef] [PubMed]

22. Nakahara, S.; Oka, N.; Raz, A. On the role of galectin-3 in cancer apoptosis. Apoptosis 2005, 10, $267-275$. [CrossRef] [PubMed]

23. Akahani, S.; NangiaMakker, P.; Inohara, H.; Kim, H.R.C.; Raz, A. Galectin-3: A novel antiapoptotic molecule with a functional BH1 (NWGR) domain of Bcl-2 family. Cancer Res. 1997, 57, 5272-5276. [PubMed]

24. Nakahara, S.; Raz, A. Regulation of cancer-related gene expression by galectin-3 and the molecular mechanism of its nuclear import pathway. Cancer Metastasis Rev. 2007, 26, 605-610. [CrossRef] 
25. Zhang, L.; Wang, P.; Qin, Y.; Cong, Q.; Shao, C.; Du, Z.; Ni, X.; Li, P.; Ding, K. RN1, a novel galectin-3 inhibitor, inhibits pancreatic cancer cell growth in vitro and in vivo via blocking galectin-3 associated signaling pathways. Oncogene 2017, 36, 1297-1308. [CrossRef]

26. Dong, R.; Zhang, M.; Hu, Q.Y.; Zheng, S.; Soh, A.; Zheng, Y.J.; Yuan, H. Galectin-3 as a novel biomarker for disease diagnosis and a target for therapy. Int. J. Mol. Med. 2018, 41, 599-614. [CrossRef]

27. Margadant, C.; van den Bout, I.; van Boxtel, A.L.; Thijssen, V.L.; Sonnenberg, A. Epigenetic Regulation of Galectin-3 Expression by beta 1 Integrins Promotes Cell Adhesion and Migration. J. Biol. Chem. 2012, 287, 44684-44693. [CrossRef]

28. Ramsey, J.; Butnor, K.; Peng, Z.H.; Leclair, T.; van der Velden, J.; Stein, G.; Lian, J.; Kinsey, C.M. Loss of RUNX1 is associated with aggressive lung adenocarcinomas. J. Cell. Physiol. 2018, 233, 3487-3497. [CrossRef]

29. Seguin, L.; Kato, S.; Franovic, A.; Camargo, M.F.; Lesperance, J.; Elliott, K.C.; Yebra, M.; Mielgo, A.; Lowy, A.M.; Husain, H.; et al. An integrin beta(3)-KRAS-RalB complex drives tumour stemness and resistance to EGFR inhibition. Nat. Cell Biol. 2014, 16, 457. [CrossRef]

30. Wang, L.; Guo, X.L. Molecular regulation of galectin-3 expression and therapeutic implication in cancer progression. Biomed. Pharmacother. 2016, 78, 165-171. [CrossRef]

31. Fukushi, J.; Makagiansar, I.T.; Stallcup, W.B. NG2 proteoglycan promotes endothelial cell motility and angiogenesis via engagement of galectin-3 and alpha 3 beta 1 integrin. Mol. Biol. Cell 2004, 15, 3580-3590. [CrossRef] [PubMed]

32. Wang, D.C.; Wang, W.; Zhu, B.J.; Wang, X.D. Lung Cancer Heterogeneity and New Strategies for Drug Therapy. Annu. Rev. Pharmacol. Toxicol. 2018, 58, 531-546. [CrossRef] [PubMed]

33. Kim, D.W.; Cochran, B.H. Extracellular signal-regulated kinase binds to TFII-I and regulates its activation of the c-fos promoter. Mol. Cell. Biol. 2000, 20, 1140-1148. [CrossRef]

34. Xu, Y.; Liu, L.; Qiu, X.; Jiang, L.; Huang, B.; Li, H.; Li, Z.; Luo, W.; Wang, E. CCL21/CCR7 promotes G2/M phase progression via the ERK pathway in human non-small cell lung cancer cells. PLoS ONE 2011, 6, e21119. [CrossRef] [PubMed]

35. Shaul, Y.D.; Seger, R. The MEK/ERK cascade: From signaling specificity to diverse functions. Biochim. Biophys. Acta 2007, 1773, 1213-1226. [CrossRef] [PubMed]

36. Song, S.M.; Ji, B.A.; Ramachandran, V.; Wang, H.M.; Hafley, M.; Logsdon, C.; Bresalier, R.S. Overexpressed Galectin-3 in Pancreatic Cancer Induces Cell Proliferation and Invasion by Binding Ras and Activating Ras Signaling. PloS ONE 2012, 7, e42699. [CrossRef]

37. Ho, M.Y.; Liang, C.M.; Liang, S.M. MIG-7 and phosphorylated prohibitin coordinately regulate lung cancer invasion/metastasis. Oncotarget 2015, 6, 381-393. [CrossRef]

38. Dumic, J.; Dabelic, S.; Flogel, M. Galectin-3: An open-ended story. Biochim. Biophys. Acta Gen. Subj. 2006, 1760, 616-635. [CrossRef]

(C) 2019 by the authors. Licensee MDPI, Basel, Switzerland. This article is an open access article distributed under the terms and conditions of the Creative Commons Attribution (CC BY) license (http://creativecommons.org/licenses/by/4.0/). 